\title{
Frecuencia y características clínicas de las cefaleas primarias en el consultorio de neurología del Hospital Nacional Almanzor Agui- naga Asenjo, año 2016-2017
}

\author{
Willie Franklin Ruiz-Cabrera ${ }^{1 a}$, Víctor Soto-Cáceres ${ }^{2 b}$
}

\section{RESUMEN}

Objetivo: Identificar la frecuencia y las características clínicas de las cefaleas primarias clasificadas en el consultorio de Neurología del Hospital Nacional Almanzor Aguinaga Asenjo. Material y Métodos: Estudio descriptivo transversal, realizado en los pacientes atendidos en el consultorio externo de Neurología del Hospital Nacional Almanzor Aguinaga Asenjo en el periodo noviembre 2016 a diciembre 2017. Se usó una encuesta validada para determinar: características clínicas y la frecuencia de las cefaleas primarias en una población adulta. Resultados: Se observó $58 \%$ de cefalea tensional, seguida de la migraña con $41,1 \%$ y la cefalea en racimos con $0,9 \%$. Las características clínicas más importantes fueron el dolor tipo opresivo (56,3\%), localización a nivel occipital $(44,2 \%)$ e intensidad incapacitante $(48,2 \%)$. El $62,5 \%$ de pacientes manifestaron que la cefalea primaria interfería en las actividades de la vida diaria. Conclusiones: La cefalea tensional y migraña predominan como cefaleas primarias en la consulta externa de Neurología del Hospital Nacional Almanzor Aguinaga Asenjo. La de tipo tensional se caracteriza principalmente por un dolor tipo opresivo y de localización occipital, mientras que la migraña, de tipo pulsátil y localización unilateral..

Palabras clave: Cefaleas primarias, Cefalea de tipo tensional, Migraña sin aura (DeCS-BIREME).

\footnotetext{
1.Universidad San Martín de Porres-Filial Norte, Lambayeque, Perú

a.Médico cirujano

${ }^{\mathrm{b}}$ Médico especialista en medicina interna y epidemiología
} 


\section{Frequency and the clinical characteristics of the primary headaches classified in the doctor's office of Neurology of the National Hospital Almanzor Aguinaga Asenjo, 2016-2017}

\section{ABSTRACT}

Objective: To identify the frequency and clinical characteristics of the primary headaches classified in the Neurology clinic of the Hospital Nacional Almanzor Aguinaga Asenjo. Material and Methods: A cross-sectional descriptive study was carried out in the patients attended in the external neurology clinic of the Hospital Nacional Almanzor Aguinaga Asenjo, from November 2016 to December 2017. A validated survey was used to determine: clinical characteristics and frequency of headaches primary in an adult population. Results: $58 \%$ of tension headache was observed, followed by migraine with $41.1 \%$ and cluster headache with $0.9 \%$. The most important clinical features were oppressive type pain $(56.3 \%)$, occipital localization $(44.2 \%)$ and disabling intensity (48.2\%). $62.5 \%$ of patients said that primary headache interfered with the activities of daily life. Conclusions: Tension headache and migraine predominate as primary headaches in the outpatient clinic of Neurology of the Hospital Nacional Almanzor Aguinaga Asenjo. The tension type is characterized mainly by oppressive type pain and occipital localization, while migraine, pulsatile type and unilateral location.

Keywords: Headache Disorders Primary, migraine without Aura y Tension-Type Headache (Source: MeSH-NLM).

\section{INTRODUCCIÓN}

Según la Organización Mundial de la Salud (OMS), las cefaleas son uno de los trastornos más comunes del sistema nervioso. Las cefaleas se caracterizan por un dolor de cabeza recurrente, se acompañan de problemas personales y sociales; provocan la discapacidad, deterioro de la calidad de vida y pérdidas económicas. Se calcula una prevalencia mundial de aproximadamente $50 \%$ en adultos (al menos una vez en el último año) ${ }^{(1)}$.

Según la sociedad internacional de cefalea (del inglés, International Headache Society), existe una clasificación que determina si una cefalea es primaria o secundaria ${ }^{(2)}$. En la cefalea primaria no existe una lesión que explique la génesis del dolor y, sin embargo, son de gran intensidad y recurrentes ${ }^{(3)}$. En la cefalea secundaria existe una causa de base, que puede ser de etología neurológica, como tumores o malformaciones vasculares, así como también de causa infecciosa, sistémica o metabólica ${ }^{(4,5)}$.

Las cefaleas primarias representan un problema de salud en las personas, las cuales muchas veces han sido subestimadas por aquellos que la padecen, y por lo tanto no han tenido un diagnóstico y tratamiento adecuado. Debido a esto, reconocer que un paciente padece cualquier tipo de cefalea primaria (por ejemplo, migraña), ayudaría a descartar cualquier otro tipo de problema neurológico y evitaría gastos tanto en el diagnóstico como en su tratamiento ${ }^{(6-7)}$.

En la región Lambayeque aún se desconoce la frecuencia y características clínicas de cefaleas primarias, por lo que se cree conveniente la aplicación de este proyecto para mejorar la problemática en torno a la frecuencia de cefaleas primarias y a la vez tener un referente acerca de dicho tema que serviría de apoyo para futuras investigaciones.

El objetivo de este estudio fue identificar la frecuencia y las características clínicas de las cefaleas primarias clasificadas en el consultorio de Neurología del Hospital Nacional Almanzor Aguinaga Asenjo, Lambayeque, Perú, en el periodo 2016 a 2017.

\section{MATERIAL Y MÉTODOS}

Tipo y diseño de la investigación

Se realizó un estudio descriptivo transversal.

\section{Población y muestra}

La población fue constituida por los pacientes que fueron atendidos en el consultorio externo de Neurología del Hospital Nacional Almanzor Aguinaga Asenjo en el periodo noviembre 2016 - noviembre 2017, cuyo diagnóstico era cualquier tipo de cefalea primaria (migraña, cefalea tensional o cefalea en racimos), la cual fue establecida por el especialista, durante el periodo 2016-2017.

La muestra fue determinada considerando una población de 799 pacientes, en consultorio externo de Neurología del Hospital Nacional Almanzor Aguinaga Asenjo durante el año 2015, luego del diagnóstico hecho en consulta externa por el especialista, con una proporción esperada de $72 \%$ (8), nivel de confianza de $95 \%$ y con una precisión de $5 \%$, se obtuvo 224 pacientes como tamaño de muestra, según el cálculo realizado en el programa EPIDAT 3. 1. Ante pérdidas como el rechazo de tres pacientes en participar en el estudio y ocho pacientes que presentaron criterios de exclusión, se agrega el $5 \%$ adicional al tamaño de muestra que equivale a 11 pacientes más por pérdidas durante la ejecución. El tipo de muestreo fue por conveniencia, ya que todos los pacientes nuevos con el diagnóstico de cefalea ingresaron al estudio hasta completar la muestra.

Se consideró como criterios de inclusión a aquellos pacien 
tes que acudieron al consultorio por cefalea primaria y que firmaron el consentimiento informado. Se excluyeron a aquellos pacientes que no pueden brindar información por alguna limitación física y a los que hayan sido evaluados en una consulta anterior.

\section{Técnicas e instrumentos de recolección de datos}

El instrumento que se utilizó fue una encuesta tipo cuestionario la cual estuvo constituida por 20 preguntas que comprenden las variables sociodemográficas, antecedentes patológicos y familiares de cefalea, localización y carácter de la cefalea, además de síntomas acompañantes y factores precipitantes más frecuentes. También se consideró el tratamiento recibido, estuvo basado en un estudio anterior ${ }^{(8)}$. El cuestionario fue validado por juicio de tres expertos, especialistas del mismo hospital.

\section{Análisis de datos}

Los datos obtenidos fueron digitados en el programa SPSS Statistics 22, en el que se aplicó frecuencias absolutas y relativas para la obtención de tablas estadísticas.

\section{Consideraciones éticas}

Para la ejecución de esta investigación, se solicitó el permiso del Comité de investigación y ética del Hospital Nacional Almanzor Aguinaga Asenjo. Se solicitó consentimiento infor mado a los pacientes y se salvaguardó la confidencialidad y anonimato de los pacientes encuestados, usando códigos.

\section{RESULTADOS}

La muestra presentó una edad predominante de 41 a 59 años, predominio femenino, instrucción superior y ama de casa (Tabla 01).

La cefalea más frecuente fue la tensional con $58 \%$, seguido por la migraña con $41 \%$ (Tabla 02), las características clínicas más frecuentes fue dolor opresivo $56,3 \%$ occipital $44,2 \%$, incapacitante $48,2 \%$ e interfiere $62,5 \%$ (Tabla 03).

La mayoría de cefaleas tipo migraña son unilaterales $92,4 \%$ mientras que las tensionales son occipitales $74,6 \%$, y bilaterales $9,2 \%$ (Tabla 04 ).

\section{DISCUSIÓN}

Los resultados obtenidos permiten afirmar que la cefalea tensional $(58 \%)$ es la más predominante de todas las cefaleas primarias en el presente estudio, seguida de la migraña $(41,1 \%)$. Sin embargo en el año anterior 2015 difiere con la base de datos obtenida del Hospital Almanzor Aguinaga Asenjo, en la que las cefaleas primarias en el año 2015 demuestran que la migraña ocupo el primer lugar con $51,4 \%$ seguido de la cefalea tensional $47,9 \%$.Existen varios estudios en los que predominó la migraña, dos de ellos realizados en

Tabla 1. Características de los pacientes con cefalea atendidos en el consultorio de Neurología del Hospital Nacional Almanzor Aguinaga Asenjo en el periodo $2016-2017$.

\begin{tabular}{lcc}
\hline Características de los pacientes & N & $\%$ \\
\hline Edad (años) & 52 & 23,2 \\
[20 - 40] & 98 & 43,8 \\
[41-59] & 74 & 33,0 \\
[60 a más] & & \\
Sexo & 42 & 18,8 \\
Masculino & 182 & 81,3 \\
Femenino & & \\
Grado de instrucción & 2 & 0,9 \\
Sin Instrucción & 35 & 15,6 \\
Primaria & 58 & 25,9 \\
Secundaria completa & 51 & 22,8 \\
Superior Técnica & 78 & 34,8 \\
Superior Universitaria & & \\
Ocupación & 31 & 13,8 \\
Profesor & 47 & 21,0 \\
Comerciante & 35 & 15,6 \\
Chofer & 12 & 5,4 \\
Personal de salud & 84 & 37,5 \\
Ama de casa & 15 & 6,7 \\
Otros & & \\
\hline \hline
\end{tabular}


Tabla 2. Frecuencia según el tipo de cefalea primaria clasificada en el consultorio de Neurología del Hospital Nacional Almanzor Aguinaga Asenjo en el periodo $2016-2017$.

\begin{tabular}{lcc}
\hline Tipo de cefalea primaria & N & $\%$ \\
\hline Migraña & 92 & 41,1 \\
Cefalea tensional & 130 & 58,0 \\
Cefalea en racimos & 2 & 0,9 \\
Total & 224 & 100 \\
\hline \hline
\end{tabular}

Tabla 3. Características clínicas de las cefaleas primarias clasificadas en el consultorio de Neurología del Hospital Nacional Almanzor Aguinaga Asenjo en el periodo 2016 - 2017.

\begin{tabular}{|c|c|c|}
\hline Características clínicas & $\mathbf{N}$ & $\%$ \\
\hline \multicolumn{3}{|l|}{ Tipo de dolor } \\
\hline Punzante & 8 & 3,6 \\
\hline Pulsátil & 89 & 39,7 \\
\hline Opresivo & 126 & 56,3 \\
\hline Calambre & 1 & 0,4 \\
\hline \multicolumn{3}{|l|}{ Localización del dolor } \\
\hline Unilateral & 92 & 41,1 \\
\hline Bilateral & 31 & 13,8 \\
\hline Occipital & 99 & 44,2 \\
\hline Región Orbitaria & 2 & 0,9 \\
\hline \multicolumn{3}{|l|}{ Intensidad del dolor } \\
\hline Leve & 14 & 6,3 \\
\hline Moderado & 102 & 45,5 \\
\hline Incapacitante & 108 & 48,2 \\
\hline \multicolumn{3}{|l|}{ Síntomas asociados } \\
\hline Náuseas & 35 & 15,6 \\
\hline Vómitos & 28 & 12,5 \\
\hline Fotofobia & 34 & 15,2 \\
\hline Sonofobia & 12 & 5,4 \\
\hline Enrojecimiento Ocular & 1 & 0,4 \\
\hline Fatiga & 23 & 10,3 \\
\hline Mareo & 28 & 12,5 \\
\hline Dolor en la nuca & 63 & 28,1 \\
\hline \multicolumn{3}{|l|}{ Interferencia del dolor } \\
\hline Interfiere & 140 & 62,5 \\
\hline No interfiere & 84 & 37,5 \\
\hline
\end{tabular}


Tabla 4. Localización del dolor de cabeza, en referencia al tipo de cefalea primaria que presentan los pacientes atendidos en el consultorio de Neurología, en el Hospital Almanzor Aguinaga Asenjo en el periodo 2016 - 2017.

\begin{tabular}{lcccccc}
\hline Tipo de Cefalea & \multicolumn{2}{c}{ Migraña } & \multicolumn{2}{c}{ Cefalea Tensional } & \multicolumn{2}{c}{ Cefalea en Racimos } \\
\hline & $\mathbf{N}$ & $\%$ & $\mathbf{N}$ & $\%$ & $\mathbf{N}$ & $\%$ \\
Unilateral & 85 & 37,9 & 7 & 3,1 & 0 & 0,0 \\
Bilateral & 6 & 2,7 & 25 & 11,2 & 0 & 0,0 \\
Occipital & 1 & 0,5 & 97 & 43,3 & 1 & 0,45 \\
Región orbitaria & 0 & 0,0 & 1 & 0,4 & 1 & 0,45 \\
Total & 92 & 41,1 & 130 & 58,0 & 2 & 0,9 \\
\hline \hline
\end{tabular}

Valladolid-España, uno por Pedraza M.I que analizó las características clínicas de las cefaleas en pacientes jóvenes, en la que $583(77,2 \%)$ pacientes presentaron migraña" (9).

Otro estudio que analizó las características demográficas de los 2000 primeros pacientes del registro de una consulta monográfica de cefaleas (CMC), y la incidencia de los diferentes tipos de cefaleas codificadas según los criterios de la clasificación internacional de cefaleas, II edición (CIC-II) en el cual, el grupo más representado era la migraña con un $53 \%{ }^{(7)}$. Un tercer estudio realizado en Colombia, observó que $56.1 \%$ presentaban migraña ${ }^{(10)}$.

Un estudio realizado por Ruiz M. en Valladolid-España en el año 2013 refiere que, $104(28,7 \%)$ pacientes presentaron cefalea tensional y $86(23,8 \%)$ de migraña ${ }^{(11)}$. Una frecuencia similar a lo observado en este estudio, a pesar de que su muestra fue sólo en pacientes mayores de 65 años y la nuestra abarcó un mayor rango de edades.

Galán-Rodas E, en el año 2010 realizó un estudio en una población rural de Cajamarca-Perú, donde determinó que la cefalea tensional $(32,27 \%)$, también fue la predominante, seguida por la migraña $(11,98 \%)$ y la cefalea en racimos $(0.24 \%)^{(8)}$.

En cuanto a las principales características clínicas, la semejanza de lo encontrado en este estudio con el de Cajamarca, abarca en que el dolor tipo opresivo $(62,2 \%)$ y pulsátil $(24,7)$ fueron los más predominantes, así como la interferencia del dolor $(54,1 \%)$ en las actividades diarias ${ }^{(8)}$. Si nos basamos

en sus menores resultados, una causa sería la ausencia de especialistas en Neurología al momento del diagnóstico, así mismo porque el Hospital Nacional Almanzor Aguinaga Asenjo atiende pacientes referidos de diversos centros de salud.

Lo más llamativo en el presente estudio fue que la cefalea primaria sí interfería en las actividades de la vida diaria con un $62,5 \%$ de los casos, lo que concuerda con lo referido por la OMS ${ }^{(1)}$ cuando relaciona a la cefalea con el deterioro de la calidad de vida y a las pérdidas económicas por ausentismo laboral. Por otro lado, nuevamente en el estudio de Galán-Rodas E, el 54,1\% de pacientes refirió la interferencia del dolor sus actividades diarias, una cifra similar a este estudio ${ }^{(8)}$

Por otra parte, las edades en las que tiene predominio la cefalea primaria según el presente estudio abarcan desde 41 a 59 años $(43,8 \%$ ) y especialmente en pacientes de sexo femenino $(81,3 \%)$; estos resultados fueron similares a los observados en Cuba, donde la población afectada era también femenina $(71,4 \%)$ y cuyas edades referidas eran de 50 a 59 años $(31,1 \%)^{(5)}$. Además, es importante remarcar en su estudio, la frecuencia en cuanto a sus tipos de cefalea primaria, ya que nos permitirá tener más conocimiento acerca de que la cefalea tensional sigue siendo la más frecuente, seguida de la migraña, todo ello también mencionado tanto en otro estudio y en la literatura ${ }^{(10,12,13)}$ como en el portal web de la Organización Mundial de la salud (OMS) ${ }^{(1)}$.

Se concluye que La cefalea tensional predomina como cefalea primaria en la consulta externa de Neurología del Hospital Nacional Almanzor Aguinaga Asenjo y sus características clínicas son: Un dolor tipo opresivo de localización occipital.

Fuentes de financiamiento: autofinanciado.

Conflictos de interés: los autores declaran no tener conflictos de intereses, ni se recibió financiamiento.

\section{REFERENCIAS BIBLIOGRÁFICAS}

1. OMS | Cefaleas [Internet]. WHO. 2016 [citado 20 de abril de 2016]. Disponible en: http://www.who.int/mediacentre/factsheets/fs277/es/

2. Comité de Clasificación de las Cefaleas. III Edición de la Clasificación Internacional de las Cefaleas. Ed Beta. 2013;370p.

3. Gómez-Otálvaro. Cefalea: Más que un simple dolor. Rev Mex Neurocienc Noviembre-Diciembre. 2015; 16(6):41-53.

4. Cid JML. Cefaleas, evaluación y manejo inicial. Rev Médica Clínica Las Condes. 2014; 25(4):651-7.

5. Quesada-Vazquez AJ, Contreras-Maure LJ, Alvarez-Aliaga A, Traba-Ta- 
mayo ER. Prevalencia de cefaleas primarias en una población rural cubana. Rev Neurol. 2009; 49(3):131-5.

6. Visens LS. Actualización en la prevención y Tratamiento de la Migraña. Med B Aires. 2014; 74(2):147-157.

7. Pedraza Hueso MI, Ruíz Piñero M, Martínez Velasco E, Juanatey García A, Guerrero Peral AL. Cefalea en jóvenes: características clínicas en una serie de 651 casos. Neurología. 2017; 34(1): 22-6.

8. Galán-Rodas E, Alarcón KN, Delgado FL, Ocampo KP, Matzunaga D. Características clínicas, epidemiológicas, terapéuticas de las cefaleas primarias en una población rural de Cajamarca, 2010. Rev Cuerpo Méd Hosp Nac Almanzor Aguinaga Asenjo. 2012 ;5(3):30-3.

9. Pedraza MI, Mulero P, Ruíz M, de la Cruz C, Herrero S, Guerrero AL. Características de los 2.000 primeros pacientes registrados en una consulta monográfica de cefaleas. Neurología. 2015; 30(4):208-13.

10. Pareja J, Campo-Arias A. Prevalencia de síntomas de ansiedad en pacientes con cefalea primaria. Acta Neurol Colomb. 2006; 22(4):299303.

11. Ruiz M, Pedraza MI, de la Cruz C, Barón J, Muñoz I, Rodríguez C, et al. Cefaleas en la persona mayor: características de una serie de 262 pacientes. Neurología. 2016; 29(6):321-6

Revisión de pares: Recibido: 27/03/2019 Aceptado: 02/04/2019 